\title{
Protective role of neuregulin-1 toward doxorubicin-induced myocardial toxicity
}

\author{
Y.Q. Liu ${ }^{1}$, M. Yang ${ }^{2}$, C.H. Duan ${ }^{1}$, G.B. Su ${ }^{1}$, J.H. Wang ${ }^{1}$, Y.F. Liu ${ }^{3}$ and \\ J. Zhang ${ }^{1}$ \\ ${ }^{1}$ Department of Cardiac Surgery, \\ The First Affiliated Hospital of Xinxiang Medical University, \\ Weihui, Henan Province, China \\ ${ }^{2}$ Department of Hematology, \\ The First Affiliated Hospital of Xinxiang Medical University, \\ Weihui, Henan Province, China \\ ${ }^{3}$ Department of General Surgery, \\ The First Affiliated Hospital of Xinxiang Medical University, \\ Weihui, Henan Province, China \\ Corresponding author: J. Zhang \\ E-mail: jiezhangen@126.com
}

Genet. Mol. Res. 13 (2): 4627-4634 (2014)

Received June 14, 2013

Accepted September 18, 2013

Published June 18, 2014

DOI http://dx.doi.org/10.4238/2014.June.18.5

\begin{abstract}
The aim of this study was to investigate the role of the rat neuregulin-1 (NRG-1) protein in reducing doxorubicin (DOX)-induced myocardial toxicity and its underlying mechanism. The prokaryotic expression of the NRG-1 protein and the CCK8-determined activity of rat primary myocardial cells were evaluated under different DOX concentrations. Myocardial cells were divided into three groups: the control group, the $5 \mu \mathrm{M}$ DOX (DOX5) group, and the DOX5 + NRG-1 group. Western blotting was used to determine the $\mathrm{Na}^{+}-\mathrm{Ca}^{2+}$ exchanger (NCX-1) and cardiac myosin light-chain kinase (cMLCK) protein expression levels and real-time quantitative polymerase chain reaction methods were used to determine the mRNA expression levels. The prokaryotic expression of NRG-1 in the DOX5 group produced
\end{abstract}


toxicity in the rat myocardial cells, and cell activity was significantly restored with the addition of NRG-1. The protective effect of NRG-1 was limited at higher DOX concentrations (DOX10), and the degree of cellular activity restoration was positively correlated with NRG-1 concentration. The addition of NRG-1 to DOX5 intervention inhibited NCX-1 protein and mRNA expression, and increased cMLCK protein and mRNA expression. In conclusion, DOX-induced toxicity in rat myocardial cells could be protected by NRG-1, and the mechanism may be related to the role of NRG-1 in up-regulating the cMLCK expression level and down-regulating the NCX-1 expression level.

Key words: Neuregulin; Doxorubicin; Myosin light-chain kinase; Cardiac myosin light-chain kinase; $\mathrm{Na}^{+}-\mathrm{Ca}^{2+}$ exchanger

\section{INTRODUCTION}

Doxorubicin (DOX), an anthracycline antibiotic that is closely related to the natural product daunomycin, has been clinically used to treat a wide range of cancers, including hematological malignancies, many types of carcinoma, and soft tissue sarcomas (Dong et al., 2009). The clinical application of DOX is limited by its severe adverse effects, including nausea, vomiting, and heart arrhythmias (Ivanová et al., 2012).

Compared with its short-term toxic effects (e.g., hematopoietic toxicity, mucosal toxicity, cardiac arrhythmias, alopecia, etc.), the principal long-term cardiac toxicity of DOX has been regarded as the most severe. Multiple chemical phenomena are putatively related to cardiotoxicity, such as activation of nuclear factor-kappa B (NF-kB), apoptosis with mitochondrial release of cytochrome $\mathrm{C}$, liberation of iron from intracellular ferritin, generation of reactive oxygen species, lipid peroxidation, and inhibition of nucleic acids and its derivative protein synthesis (Minotti et al., 2004). Previous studies have attempted to find methods to alleviate the cardiac toxicity of DOX, including slow infusion of DOX.

To date, the detailed mechanism of cardiac toxicity of DOX remains unclear. Neuregulins (NRG), proteins belonging to the epidermal growth factor (EGF) protein family, play a key role in the signaling pathway of DOX-induced cardiac toxicity (Burden and Yarden, 1997). DOX treatment could significantly reduce the protein expression of NRG-1 and receptor tyrosine kinases of the EGF receptor (EGFR) protein family ErbB4 in the heart (Horie et al., 2010). Compared with wild-type mice, DOX induced more severe cardiac injury, higher mortality, and worse left ventricular dysfunction in heterozygous NRG-1 knockout mice (Liu et al., 2005).

In the present study, the recombinant NRG-1 protein was first expressed. Furthermore, the protective role of NRG-1 toward DOX-induced toxicity in rat myocardial cells was investigated.

\section{MATERIAL AND METHODS}

\section{Experimental animals}

Sprague-Dawley (SD) rats and pregnant rats were supplied by the Experimental Animal Center of Xinxiang Medical University. This study was carried out in strict accordance with the 
recommendations in the Guide for the Care and Use of Laboratory Animals of the National Institutes of Health. The animal use protocol was reviewed and approved by the Institutional Animal Care and Use Committee (IACUC) of the First Affiliated Hospital of Xinxiang Medical University.

\section{Expression and purification of NRG-1}

The Trizol method (Invitrogen, Carlsbad, CA, USA) was used to extract total RNA for reverse transcription. The primers used for polymerase chain reaction (PCR) were NRG-1F (5'-GCGGAATTCATGTCTGAGCGCAAAG-3') and NRG-1R (5'-CGCGTCGACTTATACA GCAATAGG-3'). The PCR products were digested with EcoRI and SalI, and ligated into a $\mathrm{pET} 28 \mathrm{a}(+)$ plasmid. The vector $\mathrm{pET} 28 \mathrm{a}(+)$ and host strain BL21(DE3) were maintained in the laboratory. Expression vectors were transformed into Escherichia coli strain BL21(DE3)competent cells. Transformed cells were grown at $37^{\circ} \mathrm{C}$ in Luria-Bertani medium supplemented with $50 \mu \mathrm{g} / \mathrm{mL}$ kanamycin to a cell density of $\mathrm{A}_{660 \mathrm{~nm}}=0.4-0.6$ and induced with $1 \mathrm{mM}$ isopropyl$\beta$-D-thiogalactoside (IPTG) for $2 \mathrm{~h}$ at $37^{\circ} \mathrm{C}$. Cells were then pelleted and resuspended in sample buffer and the presence of restriction enzyme fragments in eluted fractions was confirmed by denatured sodium dodecyl sulfate-polyacrylamide gel electrophoresis (SDS-PAGE) analysis. To purify the NRG-1, approximately $1 \mathrm{~L}$ cultured transformed cells was centrifuged and then pellets were resuspended in $5 \mathrm{~mL}$ phosphate-buffered saline (PBS), $\mathrm{pH} 7.4$, containing $1 \mathrm{mM}$ phenylmethylsulfonyl fluoride (PMSF), sonicated, and centrifuged at $8000 \mathrm{rpm}$ at $4{ }^{\circ} \mathrm{C}$ for 10 $\mathrm{min}$. The resulting lysates were purified using the His-Tag method according to manufacturer instructions (Novagen, Darmstadt, Germany). Purified NRG-1 was dialyzed against PBS, pH 7.4, and protein concentrations were determined by the Bradford method.

\section{Isolation and cultivation of rat primary myocardial cells}

The method used to isolate myocardial cells was modified from Yang et al. (2002). Newborn SD rats were alcohol-disinfected and their heads were cut. Their ventricles were placed in pre-chilled Dulbecco's modified Eagle's medium (DMEM) after thoracotomy. After cleaning, the DMEM was discarded and the ventricles were minced on a plastic dish. Tissues were digested in $6 \mathrm{~mL}$ pancreatin in culture bottles for $15 \mathrm{~min}$ in a $37^{\circ} \mathrm{C}$ water bath and shaken at $100 \mathrm{rpm}$. Tissues were then mixed with $3.2 \mathrm{~mL}$ PBS, $0.4 \mathrm{~mL}$ collagenase, and $0.4 \mathrm{~mL}$ pancreatin for 10 min digestion in a $37^{\circ} \mathrm{C}$ water bath at $100 \mathrm{rpm}$, and the supernatant was discarded. The supernatant was collected in a tube containing $5 \mathrm{~mL}$ bovine serum and stored at room temperature. These procedures were repeated until the tissues were completely digested. The supernatant was collected and centrifuged for $10 \mathrm{~min}$ at $1000 \mathrm{rpm}$. The precipitate was resuspended in $13 \mathrm{~mL}$ DMEM, and then transferred to an open culture dish for $45 \mathrm{~min}$ at $37^{\circ} \mathrm{C}$. The cell suspension was transferred to another culture dish after gently shaking. The cell suspension was collected and counted after repeating the process twice, and then transferred to a culture dish with the desired cell number for attached growth.

\section{The effect of DOX on cell growth}

Cells were inoculated on a 12 -well plates with $1 \times 10^{6}$ cells per well. Cell growth was determined after treatment with $0,1,2.5,5,10$, and $20 \mu \mathrm{M}$ DOX (Zhejiang Haimen Pharmaceutical Inc., Haimen, China) for $72 \mathrm{~h}$. 


\section{Determination of CCK8 activity}

Rat cells were inoculated on 96-well plates at a density of $10^{4}$ cells per well and divided into three groups: the control group, the DOX-treated group, and the DOX + NRG-1-treated group. DOX was used at $5 \mu \mathrm{M}$ (DOX5) and $10 \mu \mathrm{M}$ (DOX10) concentrations. Various concentrations of NRG-1 $(0,0.14,0.41,1.22,3.67,11,33$, and $99 \mu \mathrm{M})$ were used. After 6 days of treatment, the CCK8 activity was determined as described previously.

\section{Western blotting analysis and quantitative PCR}

The protein concentration was determined using the bicinchoninic assay method, and $5 \mu \mathrm{g}$ protein was loaded for Western blotting analysis using GADPH as the internal standard, as previously described. $\mathrm{Na}^{+}-\mathrm{Ca}^{2+}$ exchanger (NCX-1) antibody, GAPDH antibody, cardiac myosin light-chain kinase (cMLCK) antibody, and the secondary antibody were purchased from Santa Cruz Biotechnology (Santa Cruz, CA, USA). Quantitative PCR was carried out as previously described using the following primers: NCX-1F:5'-GGAAGATGATGACGACGAT3', NCX-1R: 5'-CAGCCATTCCAGTATTCAGT-3'; cMLCK-F: 5'-CACTGAGGCAGGGAG TTGA-3', cMLCK-R: 5'-GGCTTGCTGGATGAGGTT-3'; GAPDH-F: 5'-CTGGAAGAT GGTGATGGGATT-3', GAPDH-R: 5'-ATGGGGAAGGTGAAGGTCG-3'. The primers were synthesized by the GenScript Co., Ltd. (Nanjing, China).

\section{Statistical analysis}

The SPSS 13.0 software (SPSS Inc., Chicago, IL, USA) was used for statistical analysis. All data are reported as means $\pm \mathrm{SD}$. Statistical significance was determined by the Student $t$-test where $\mathrm{P}<0.05$ was considered to be statistically significant.

\section{RESULTS}

\section{Successful expression of NRG-1}

The results of electrophoresis of the recombinant plasmid and enzyme-digested fragments are shown in Figure 1A and B. The molecular weight of the expressed NRG-1 protein was $73.7 \mathrm{kDa}$ (Figure 1C), and NRG-1 was purified well as shown in Figure 1D.

\section{DOX-induced myocardial toxicity with increasing concentrations}

Myocardial cells were inoculated on 12-well plates with 1 x $10^{6}$ cells per well. After $12 \mathrm{~h}$, cells were treated with $0,1,2.5,5,10$, and $20 \mu \mathrm{M}$ DOX for $24 \mathrm{~h}$. The growth state of the cells was determined by microscopic observation (Figure 2). The cells grew well without DOX; however, when the DOX concentration increased to 1 and $2.5 \mu \mathrm{M}$, the cells grew slowly and changed in morphology. At 5, 10, and $20 \mu \mathrm{M}$ DOX, cell toxicity was evident and cells died and floated upward. 
A
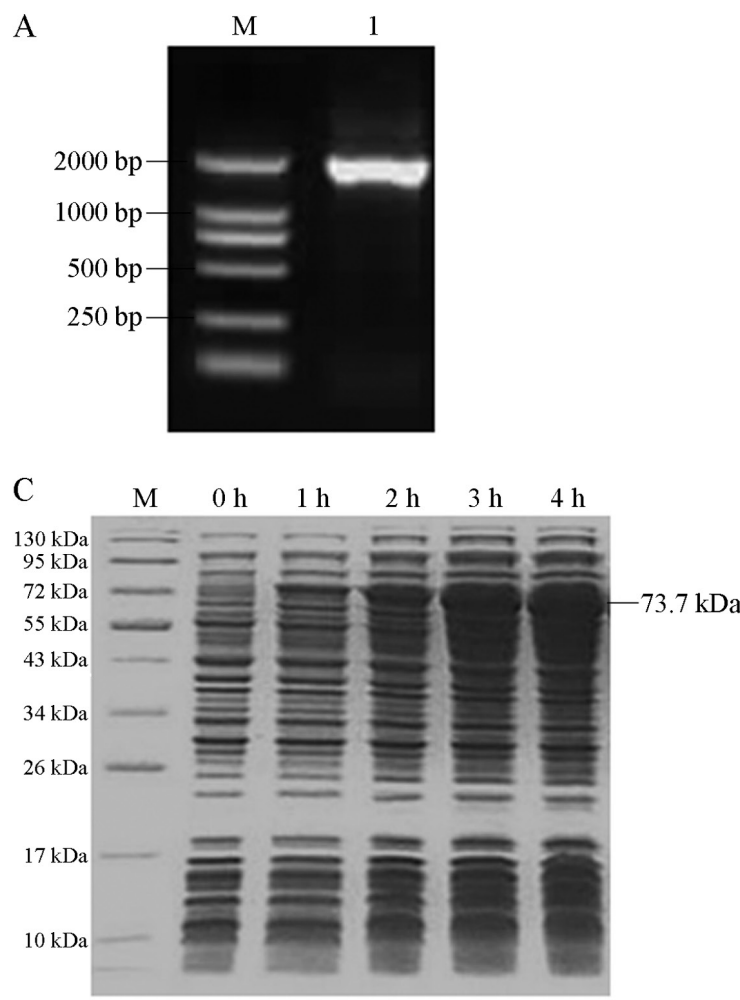

B
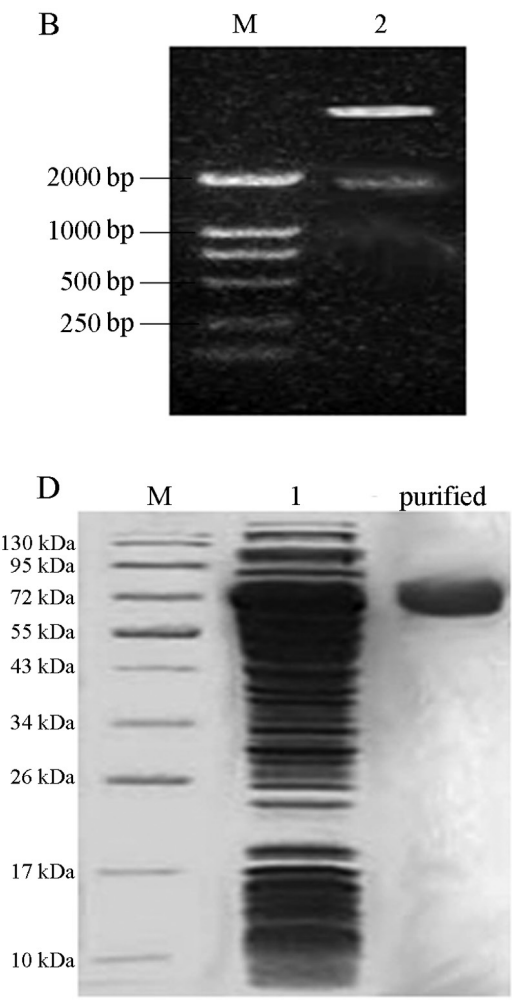

Figure 1. Agarose gel electrophoresis. A. Results of NRG-1 PCR amplification. Lane $M=$ DL2000 (100, 250, 500, 750, 1000, 2000 bp); lane 1 = NRG-1 (1929 bp). B. pET28a(+)-NRG-1 restriction enzyme digested results. Lane $M=\mathrm{DL} 2000$; lane $2=\mathrm{pET} 28 \mathrm{a}(+)-\mathrm{NRG}-1$ EcoRI and Sall restriction enzyme results. C. PET28a(+)-NRG-1 after induction of SDS-PAGE electrophoresis results. D. Ni-NTA purification (6His-Tag) SDS-PAGE electrophoresis results. Lane $M=$ low molecular weight protein marker $(10-130 \mathrm{kDa})$; lane $1=$ unpurified.

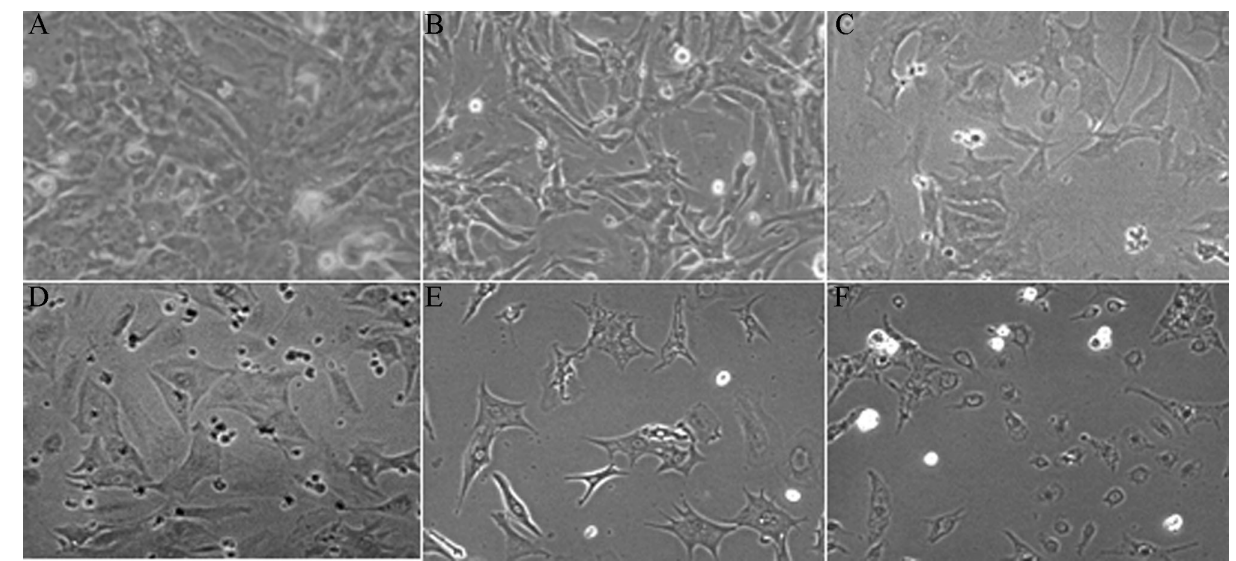

Figure 2. Primary rat myocardial cell growth state under different DOX concentration (100X). A. $0 \mu \mathrm{M}$ DOX; B. $1 \mu \mathrm{M}$ DOX; C. $25 \mu \mathrm{M}$ DOX; D. $5 \mu \mathrm{M}$ DOX; E. $10 \mu \mathrm{M}$ DOX; F. $20 \mu \mathrm{M}$ DOX. 


\section{NRG-1 could restore the activity of DOX5-treated myocardial cells}

Myocardial cells were inoculated on 12 -well plates with $1 \times 10^{6}$ cells per well. After $12 \mathrm{~h}$, the cells were treated with 5, 10, and $20 \mu \mathrm{M}$ DOX, and NRG-1 was added to each DOX treatment at different concentrations $(0,0.14,0.41,1.22,3.67,11,33$, and $99 \mu \mathrm{M})$. The cells' activity was measured after culturing for $24 \mathrm{~h}$ and the results are shown in Figure 3.

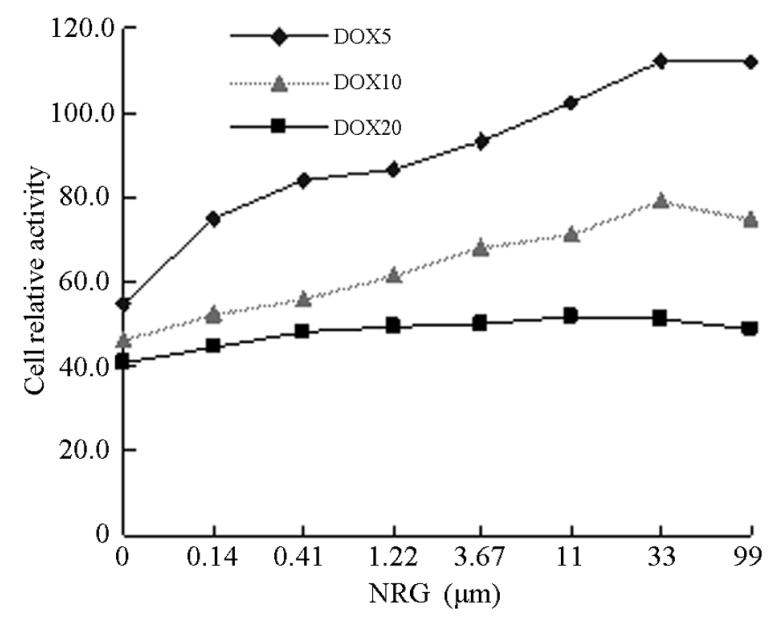

Figure 3. NRG-1 and DOX on rat myocardial cell activity of CCK8 experiment.

\section{NRG could down-regulate NCX-1 and up-regulate cMLCK expression in DOX5- induced myocardial cells}

Four groups of myocardial cells, including the control group, the DOX5 group, the DOX5 $+11 \mu \mathrm{M}$ NRG-1 group, and the $11 \mu \mathrm{M}$ NRG-1 group were cultured for $24 \mathrm{~h}$. Western blotting analysis was used to determine the expression levels of NCX-1 and cMLCK and the results are shown in Figure 4. The corresponding results obtained by fluorescence quantitative PCR are shown in Figure 5.

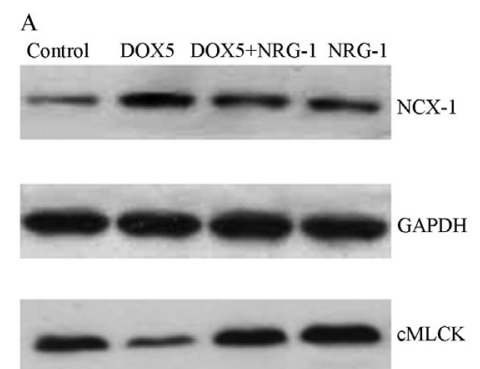

B

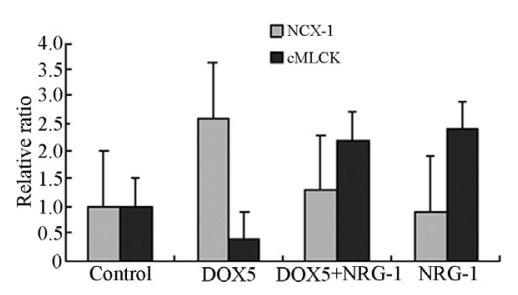

Figure 4. Analysis of Western blotting in rat myocardial cells. NCX-1 gene: $\mathrm{P}<0.05$ for the DOX5+NRG-1 group compared with the other three groups. Control, DOX5+NRG-1 and NRG-1 groups showed no significant difference $(\mathrm{P}>0.05)$. cMLCK gene: $\mathrm{P}<0.05$ for the DOX5+NRG-1 group compared with the DOX5 group. The DOX5+NRG-1 group compared with the NRG-1 group showed no significant differences; each group compared with the control group showed significant differences $(\mathrm{P}<0.05)$. 


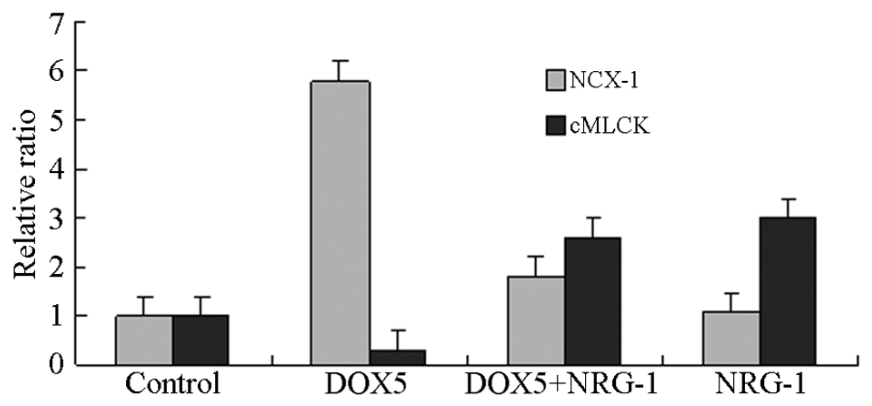

Figure 5. Analysis of rat myocardial cells by fluorescence quantitative PCR. NCX-1 gene: $\mathrm{P}<0.05$ for the DOX5+NRG-1 group compared with the other three groups. Control, DOX5+NRG-1 and NRG-1 groups were not significantly different. cMLCK gene: $\mathrm{P}<0.05$ for the DOX5+NRG-1 group compared with the DOX5 group. The DOX5+NRG-1 group compared with the NRG-1 group showed no significant differences $(\mathrm{P}>0.05)$. Each group compared with the control group showed significant differences $(\mathrm{P}<0.05)$.

\section{DISCUSSION}

The cloning of the rat NRG-1 gene, the development of the prokaryotic expression system, and the success of the nickel-nitrotetraacetic acid system for purifying the NRG-1 protein have provided a basis for further research into the protein's biological activity. Zhang et al. (1998) showed that tea polyphenols could reduce DOX-induced cardiac toxicity in rats by the removal of DOX-triggered free radicals, DOX-induced lipid peroxidation, and through the protection of superoxide dismutase and glutathione peroxidase activity. In this experiment, DOX intervention reduced primary rat myocardial cell growth activity with increasing concentration, inhibited cell growth to various degrees, and even caused irreversible changes resulting in cell damage and death. Gao et al. (2010) showed that NRG-1 could improve the myocardial structure and increase the left ventricular ejection fraction in heart failure patients, and other studies have also indicated that NRG-1 could exert favorable effects following heart failure (Gu et al., 2010; Hedhli et al., 2011; Jabbour et al., 2011; Li et al., 2011). We here investigated whether NRG-1 might also play a protective role in the DOX-induced toxicity in myocardial cells. We hypothesized that NRG-1 treatment following DOX intervention would show different degrees of recovery in myocardial cell activity in a concentration-dependent manner. The results showed that DOX5 induced toxicity to myocardial cells reducing their viability, and NRG-1 treatment had obvious restorative and recovery effects on DOX10-induced toxicity and cell viability. However, when the DOX concentration was too high, the protective effect of NGR-1 was limited or absent, because the cells had already undergone irreversible injury or death.

MLCK is a key regulatory protein involved in smooth muscle cell contraction, and its structure consists of an $\mathrm{N}$-terminal actin binding region, a central region, a calmodulin kinase binding domain, and a C-terminal myosin binding region. cMLCK is a cardiac-specific MLCK that plays a key role in cardiac contractility (Rottbauer et al., 2006). With the continuous action of DOX, the mRNA and protein expression levels of cMLCK dropped significantly; however, under different concentrations of NRG-1, cMLCK expression increased to normal levels, and cell growth was improved. NCX-1 is an important excitable two-way ion transport protein of the cell membrane, and its main function is to discharge cell excitability during 
entry, recovering the intracellular $\mathrm{Ca}^{2+}$ level to the resting state in each discharge of $1 \mathrm{Ca}^{2+}$ and $3 \mathrm{Na}^{+}$into the cell, thus maintaining a net charge of transmembrane movement to generate a transmembrane current (Gaughan et al., 1999). Thus, expression changes of NCX-1, especially at abnormal elevation, may induce cell membrane damage resulting in membrane potential abnormalities and membrane permeability, so that a large number of $\mathrm{Na}^{+}$ions would enter the cell, thus activating the $\mathrm{Na}^{+}-\mathrm{Ca}^{2+}$ reverse switch, causing an intracellular $\mathrm{Ca}^{2+}$ overload, and thereby further increasing cell damage leading to cell death. In this study, DOX induced an increase in rat myocardial cell NCX-1 levels, which was considerably inhibited by the addition of different NRG-1 concentrations. Therefore, the addition of NRG-1 to DOX intervention produces a protective effect on DOX-induced rat myocardial cell toxicity, and its mechanism may be related to the up-regulation of cMLCK expression and the down-regulation of NCX-1 expression.

\section{REFERENCES}

Burden S and Yarden Y (1997). Neuregulins and their receptors: a versatile signaling module in organogenesis and oncogenesis. Neuron 18: 847-855.

Dong X, Liu A, Zer C, Feng J, et al. (2009). siRNA inhibition of telomerase enhances the anti-cancer effect of doxorubicin in breast cancer cells. BMC Cancer 9: 133.

Gao R, Zhang J, Cheng L, Wu X, et al. (2010). A phase II, randomized, double-blind, multicenter, based on standard therapy, placebo-controlled study of the efficacy and safety of recombinant human neuregulin-1 in patients with chronic heart failure. J. Am. Coll. Cardiol. 55: 1907-1914.

Gaughan JP, Furukawa S, Jeevanandam V, Hefner CA, et al. (1999). Sodium/calcium exchange contributes to contraction and relaxation in failed human ventricular myocytes. Am. J. Physiol. 277: H714-H724.

$\mathrm{Gu}$ X, Liu X, Xu D, Li X, et al. (2010). Cardiac functional improvement in rats with myocardial infarction by up-regulating cardiac myosin light chain kinase with neuregulin. Cardiovasc. Res. 88: 334-343.

Hedhli N, Huang Q, Kalinowski A, Palmeri M, et al. (2011). Endothelium-derived neuregulin protects the heart against ischemic injury. Circulation 123: 2254-2262.

Horie T, Ono K, Nishi H, Nagao K, et al. (2010). Acute doxorubicin cardiotoxicity is associated with miR-146a-induced inhibition of the neuregulin-ErbB pathway. Cardiovasc. Res. 87: 656-664.

Ivanová M, Dovinova I, Okruhlicova L, Tribulova N, et al. (2012). Chronic cardiotoxicity of doxorubicin involves activation of myocardial and circulating matrix metalloproteinases in rats. Acta Pharmacol. Sin. 33: 459-469.

Jabbour A, Hayward CS, Keogh AM, Kotlyar E, et al. (2011). Parenteral administration of recombinant human neuregulin-1 to patients with stable chronic heart failure produces favourable acute and chronic haemodynamic responses. Eur. J. Heart Fail. 13: 83-92.

Li B, Zheng Z, Wei Y, Wang M, et al. (2011). Therapeutic effects of neuregulin-1 in diabetic cardiomyopathy rats. Cardiovasc. Diabetol. 10: 69.

Liu FF, Stone JR, Schuldt AJ, Okoshi K, et al. (2005). Heterozygous knockout of neuregulin-1 gene in mice exacerbates doxorubicin-induced heart failure. Am. J. Physiol. Heart Circ. Physiol. 289: H660-H666.

Minotti G, Menna P, Salvatorelli E, Cairo G, et al. (2004). Anthracyclines: molecular advances and pharmacologic developments in antitumor activity and cardiotoxicity. Pharmacol. Rev. 56: 185-229.

Rottbauer W, Wessels G, Dahme T, Just S, et al. (2006). Cardiac myosin light chain-2: a novel essential component of thick-myofilament assembly and contractility of the heart. Circ. Res. 99: 323-331.

Yang YL, Liu JT, Lin R and Li DM (2002). 3,6-(2-Dimethylamino)-2 benzene and iodine six miscellaneous ring gluconate on hydrogen peroxide induced injury in cultured rat myocardial cells in rats. Chin. J. Pharmacol. Toxicol. 16: 106-109.

Zhang ZM, Geng BQ and Yong DG (1998). Effect of tea polyphenols on doxorubicin-induced cardiotoxicity in rats. Chin. Pharmaceutical J. 33: 405-409. 